Supporting Information

\title{
Development of a New Method for Synthesis of Tandem Hairpin Pyrrole-Imidazole Polyamide Probes Targeting Human Telomeres
}

Yusuke Kawamoto ${ }^{\text {a }}$, Toshikazu Bando, ${ }^{*}$, Fukumi Kamada ${ }^{\mathrm{b}}$, Yue Li ${ }^{\mathrm{a}}$, Kaori Hashiya ${ }^{\mathrm{a}}$, Kazuhiro Maeshima $^{*}$,b,c , and Hiroshi Sugiyama ${ }^{*, a, d, e}$

${ }^{\mathrm{a}}$ Department of Chemistry, Graduate School of Science, Kyoto University, Sakyo, Kyoto 606-8502, Japan

${ }^{\mathrm{b}}$ Biological Macromolecules Laboratory, Structural Biology Center, National Institute of Genetics, Mishima, Shizuoka 411-8540, Japan

${ }^{\mathrm{c}}$ Department of Genetics, School of Life Science, Graduate University for Advanced Studies (Sokendai), Mishima, Shizuoka 411-8540, Japan

${ }^{\mathrm{d}}$ Institute for Integrated Cell-Material Science (WPI-iCeMS), Kyoto University, Sakyo, Kyoto 606-8501, Japan

${ }^{\mathrm{e}}$ Core Research for Evolutional Science and Technology (CREST), Japan Science and Technology Corporation (JST), Sanbancho, Chiyoda-ku, Tokyo 102-0075, Japan 
(a)

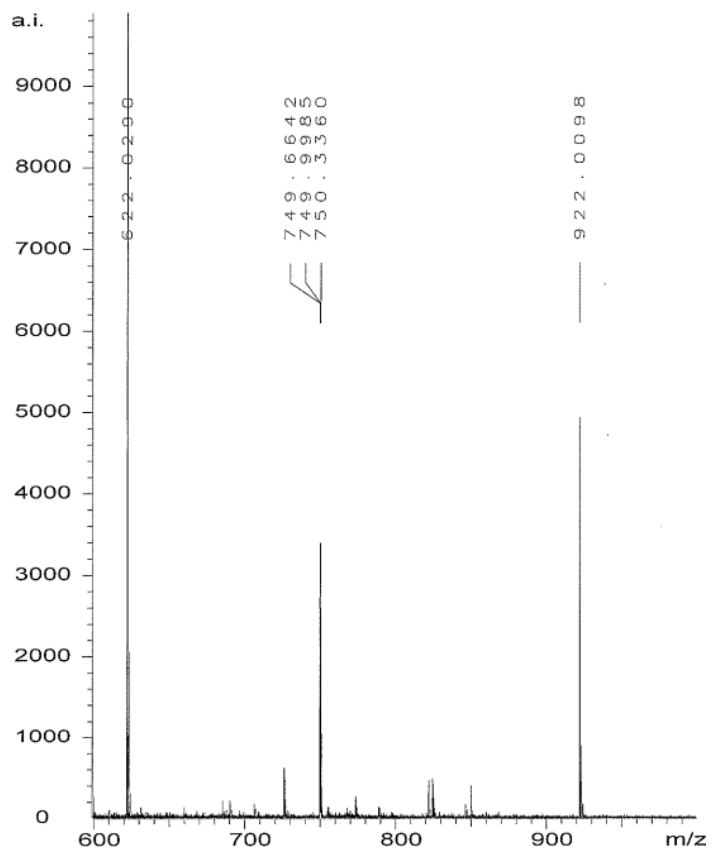

(b)

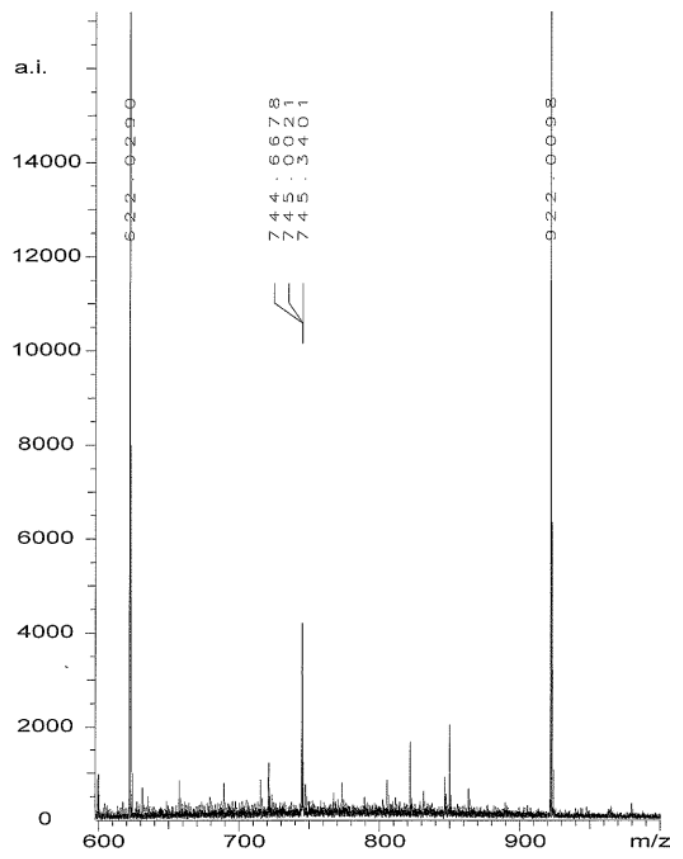

Figure S1. ESI-TOF MS spectra of the compound 10 (a) and 11 (b). 
(a)

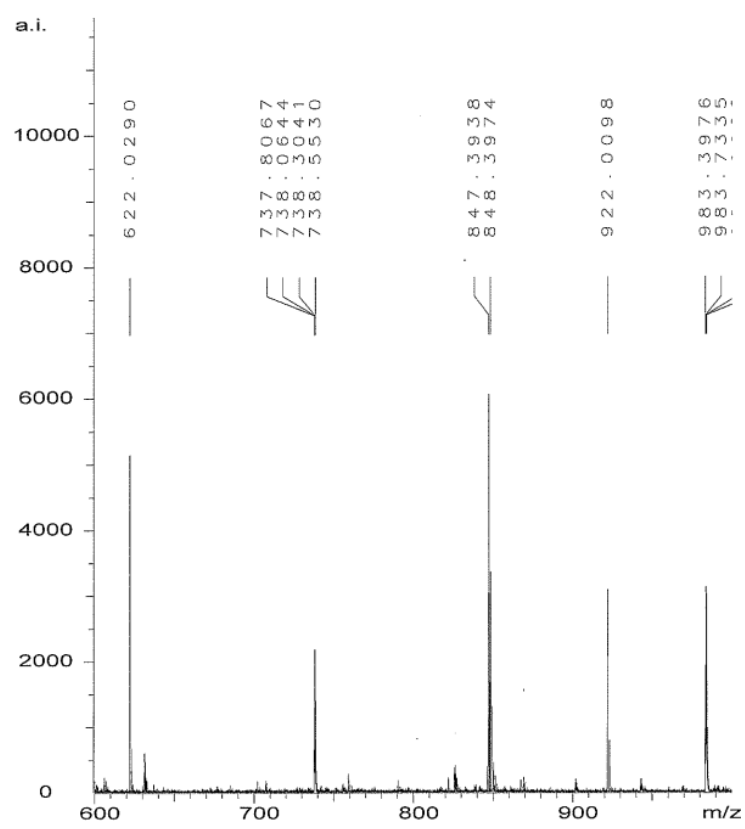

(c)

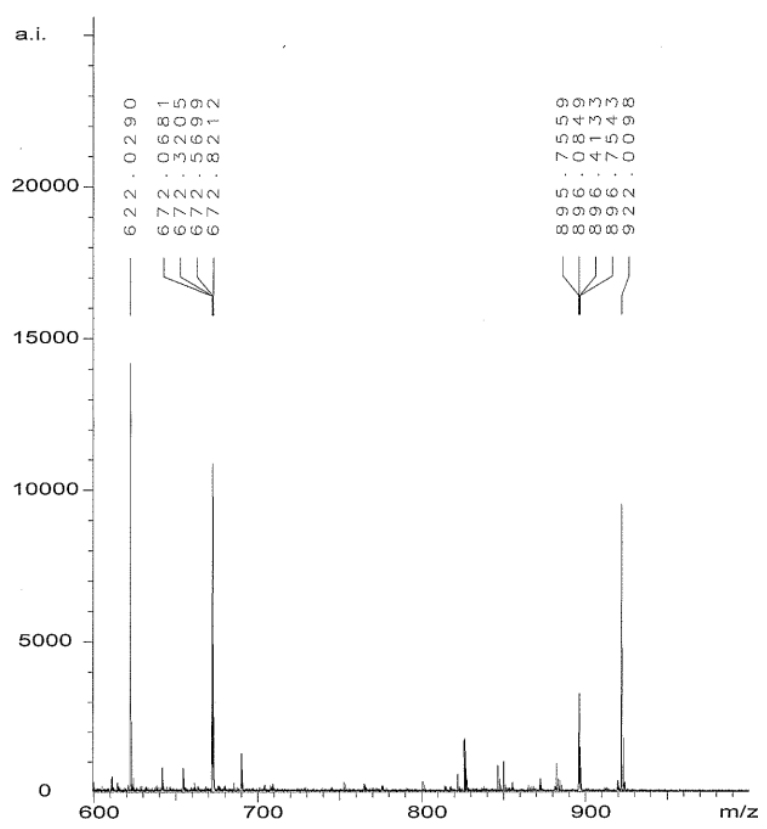

(b)

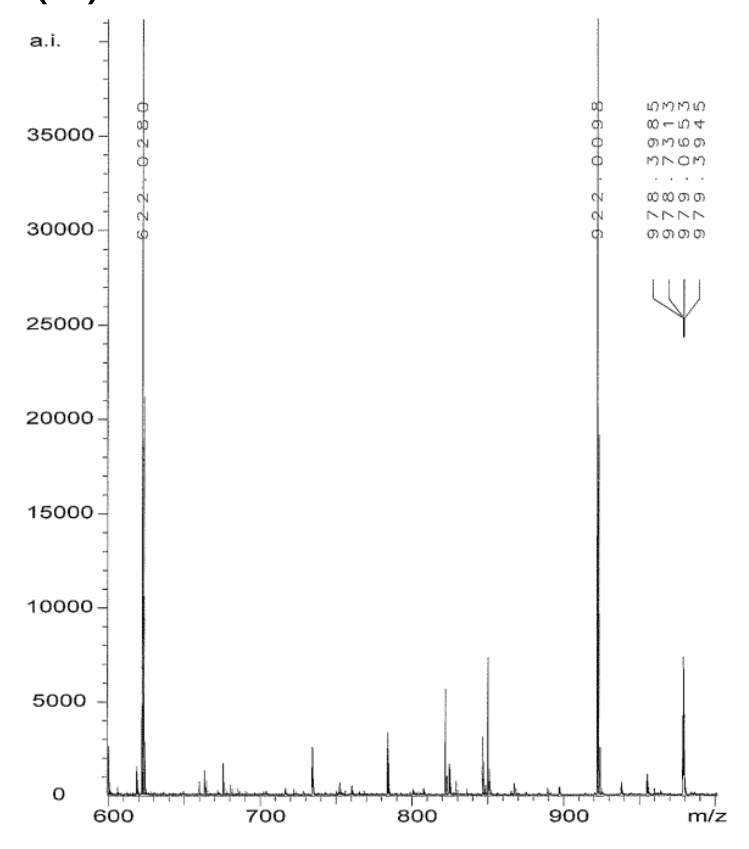

(d)

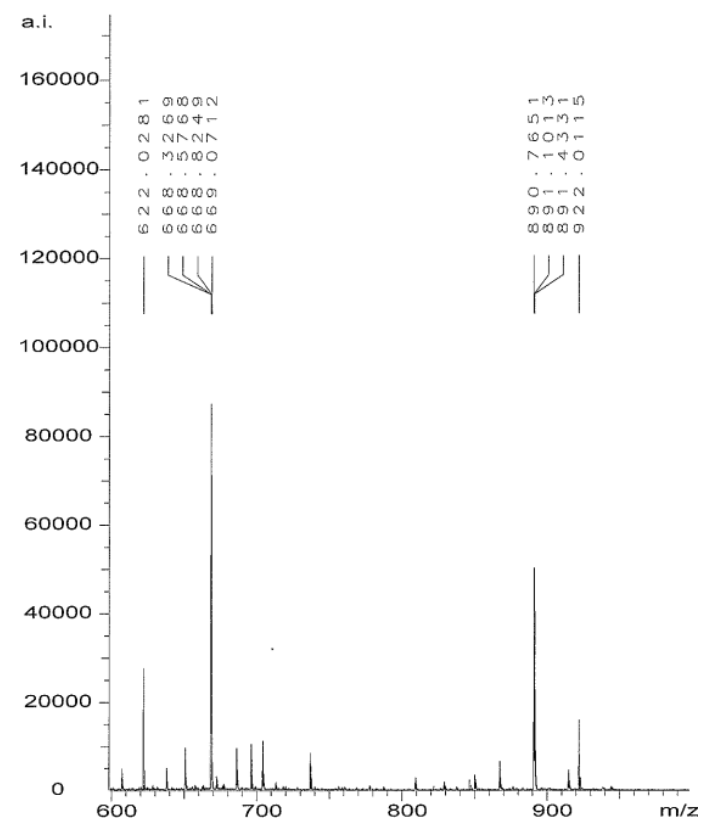

Figure S2. ESI-TOF MS spectra of the conjugate 1-4 (a: 1, b: 2, c: 3, d: 4). 
(a)

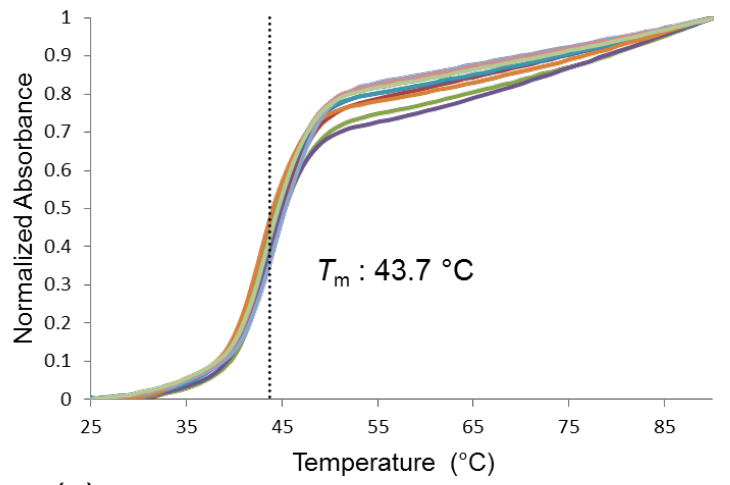

(c)

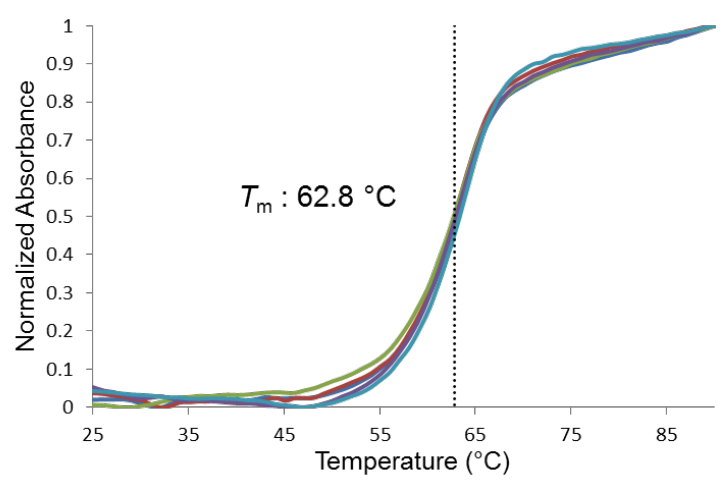

(b)

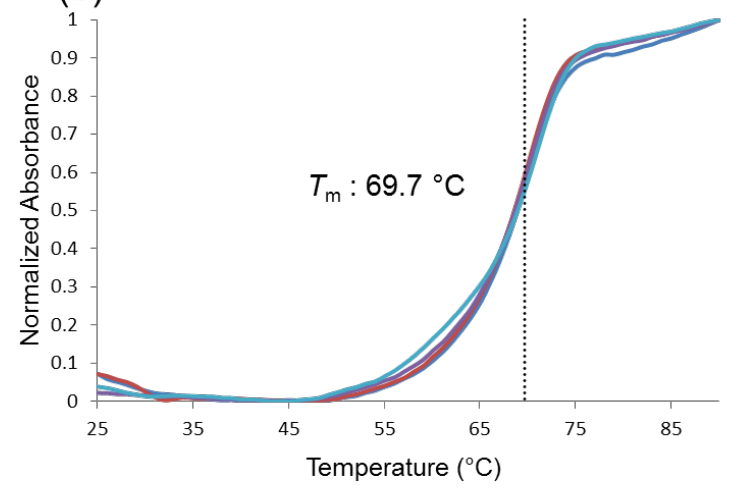

Figure S3. UV melting profiles at $260 \mathrm{~nm}$ for ODN-1/2 (a), TH59-ODN-1/2 complex (b),

Deamino TH59-ODN-1/2 (c). The pointed lines in Figure S3-6 are at $T_{\mathrm{m}}$. 
(a)

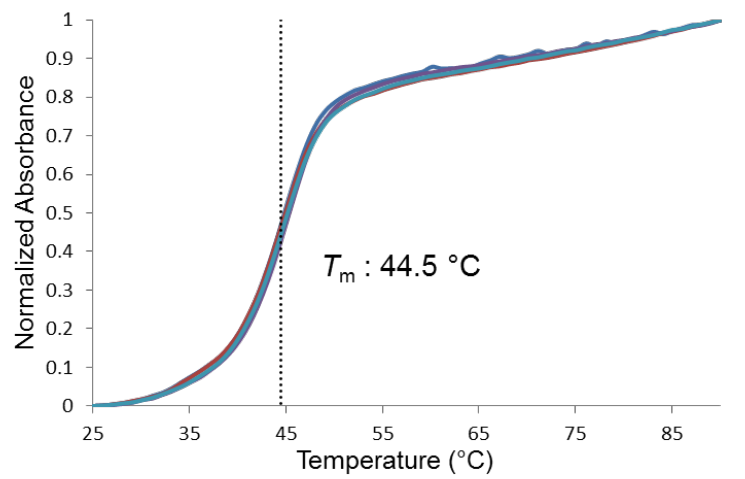

(c)

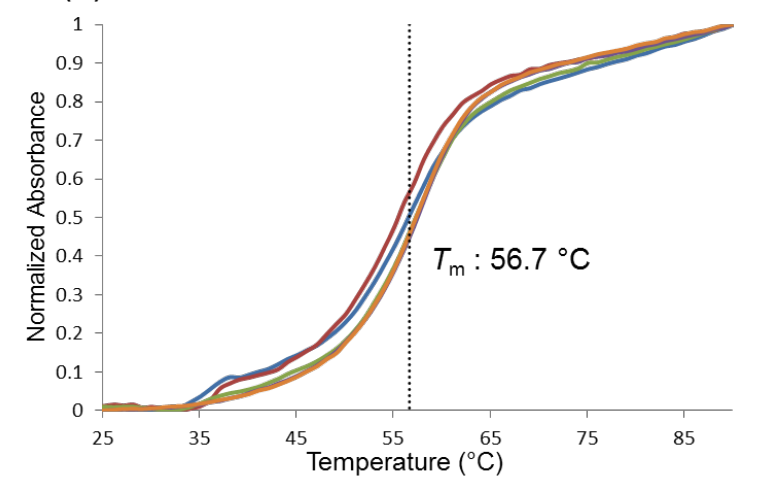

(b)

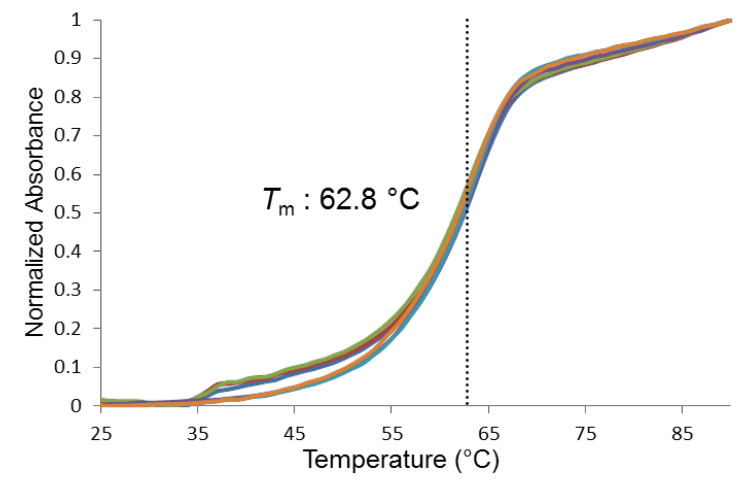

Figure S4. UV melting profiles at $260 \mathrm{~nm}$ for ODN-3/4 (a), TH59-ODN-3/4 complex (b), Deamino TH59-ODN-3/4 (c). 
(a)

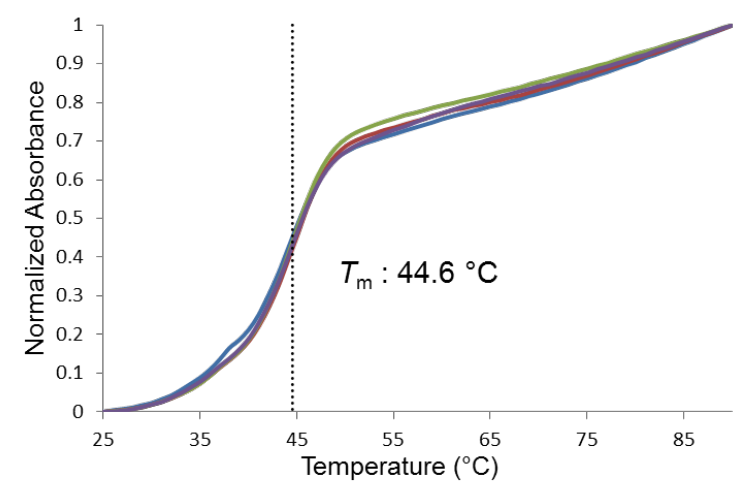

(c)

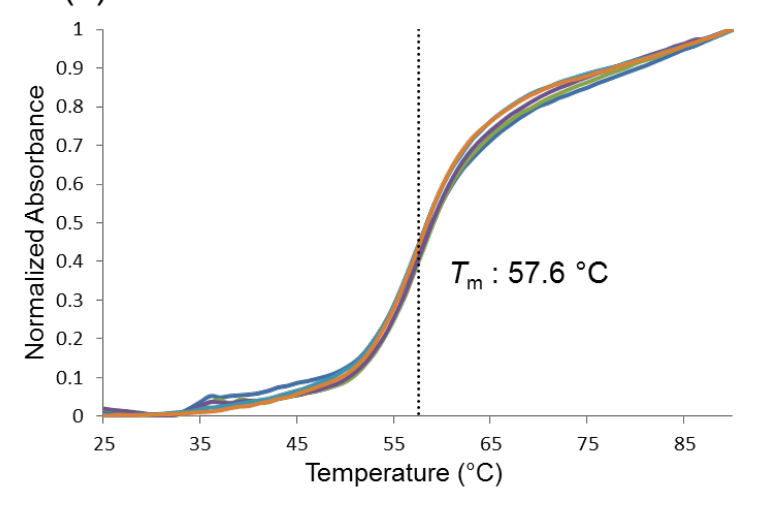

(b)

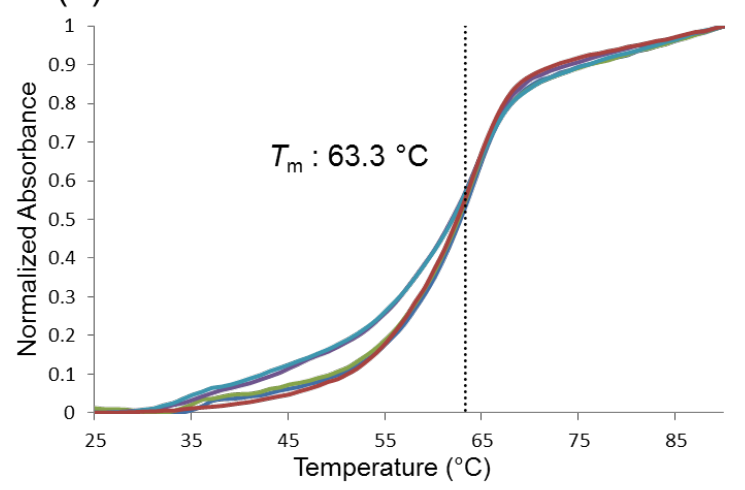

Figure S5. UV melting profiles at $260 \mathrm{~nm}$ for ODN-5/6 (a), TH59-ODN-5/6 complex (b), Deamino TH59-ODN-5/6 (c). 
(a)

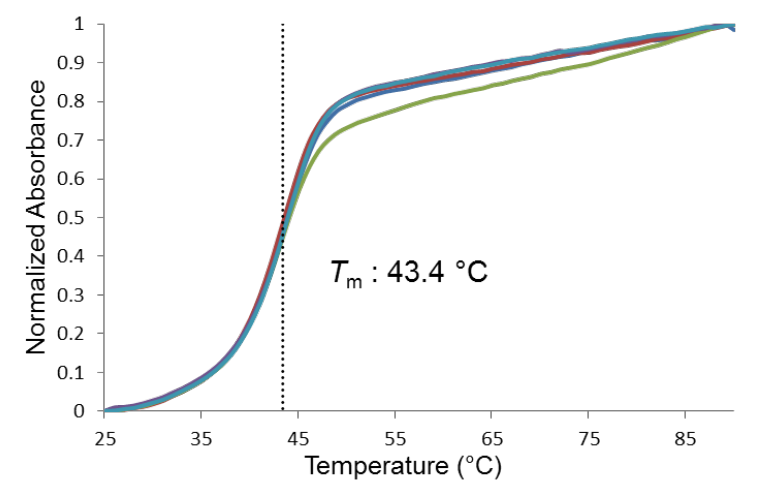

(c)

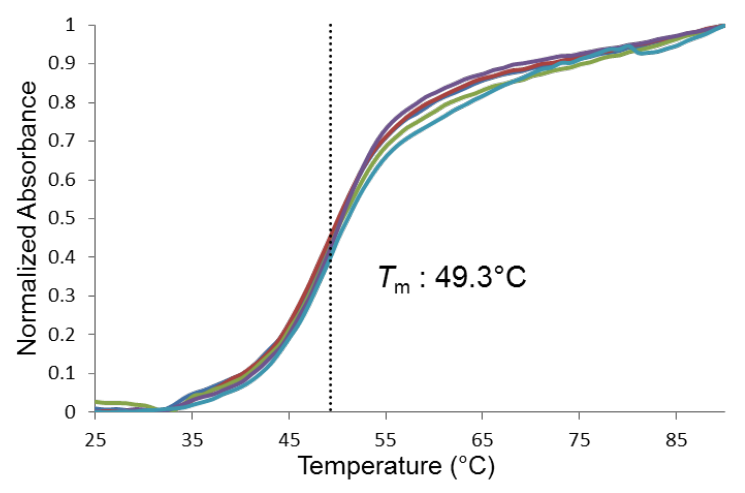

(b)

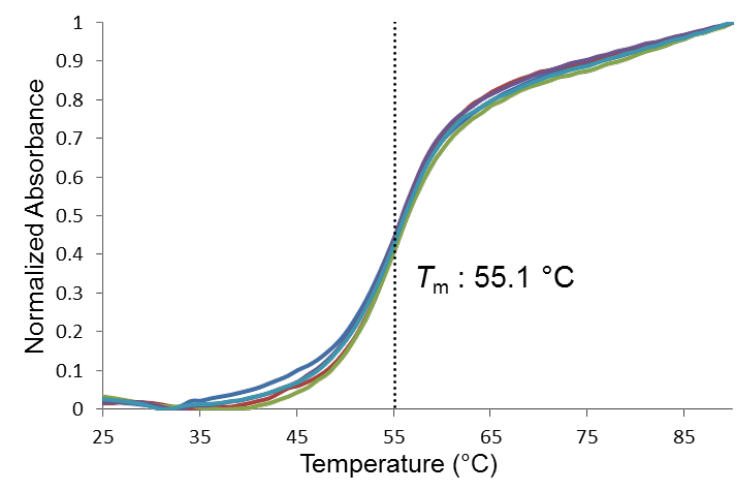

Figure S6. UV melting profiles at $260 \mathrm{~nm}$ for ODN-7/8 (a), TH59-ODN-7/8 complex (b), Deamino TH59-ODN-7/8 (c). 


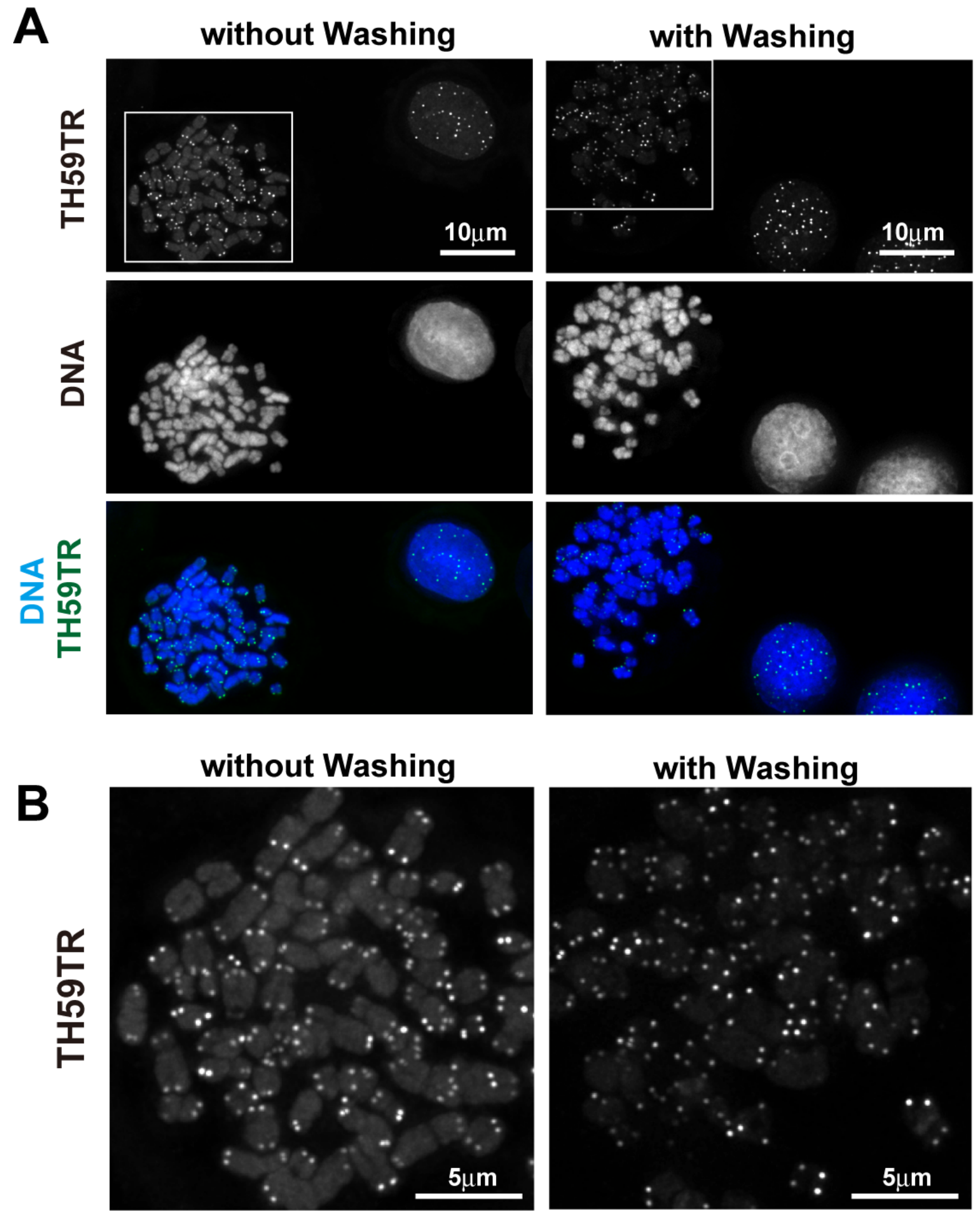


Figure S7. Effect of washing on telomere staining by TH59TR. (A) Spreads of HeLa1.3 cells were stained with TH59TR with (right) or without (left) a washing process. The preparation of cell spreads and TH59TR staining were performed as described in the "Materials and Methods". Note that the telomeric signals were detected without the washing process, but the process helped reduce the background or nonspecific PI polyamide binding. (B) Enlarged images of the regions marked in Panel A. 